\title{
Erratum to: Impact of rapid ventricular pacing during TAVI on microvascular tissue perfusion
}

\author{
Anna Selle $\cdot$ Hans R. Figulla - Markus Ferrari - Wilma Rademacher • \\ Bjoern Goebel • Ali Hamadanchi • Marcus Franz • Andrea Schlueter • \\ Thomas Lehmann · Alexander Lauten
}

Published online: 29 July 2014

(c) Springer-Verlag Berlin Heidelberg 2014

\section{Erratum to: Clin Res Cardiol \\ DOI 10.1007/s00392-014-0728-9}

Due to an editing mistake in the lines of the table some of the numbers are mixed up. Please find below the corrected Table 1.

The online version of the original article can be found under doi:10. 1007/s00392-014-0728-9.

\section{A. Selle $\cdot$ H. R. Figulla $\cdot$ W. Rademacher $\cdot$ B. Goebel .}

A. Hamadanchi · M. Franz · A. Lauten $(\square)$

Department of Internal Medicine I (Cardiology, Angiology, Pneumology), University Hospital Jena, Erlanger Allee 101, 07740 Jena, Germany

e-mail: alexander.lauten@med.uni-jena.de

\section{Ferrari}

Department of Internal Medicine I (Cardiology, Angiology, Conservative Intensive Care), Dr.-Horst-Schmidt Hospital, Ludwig-Erhard-Straße 100, 65199 Wiesbaden, Germany

\footnotetext{
A. Schlueter

Department of Anaesthesiology and Critical Care Therapy,

Friedrich-Schiller-University, Jena, Germany

T. Lehmann

Institute of Medical Statistics, Computer Sciences and Documentation, University Hospital, Jena Bachstraße 18, 07743 Jena, Germany
}

Table 1 Patient demographics and comorbidities

Population $(n=42)$

\section{Patient characteristics}

Age (years \pm SD)

$81.21 \pm 8.44$
$18 / 26$
$0.64 \pm 0.17 \mathrm{~cm}^{2}$
$4.58 \pm 0.66 \mathrm{~m} / \mathrm{s}$
$48.79 \pm 16.73 \mathrm{mmHg}$
$55.93 \pm 15.89 \%$
$28.89 \pm 14.07$
$42(100)$
$32(76.2)$
$19(45.2)$
$11(26.2)$
$10(23.8)$
$9(21.4)$
$7(16.67)$
$5(11.9)$
$3(7.14)$

Male, $n(\%)$

$\operatorname{AVA}\left(\mathrm{cm}^{2} \pm \mathrm{SD}\right)$

$V_{\max }(\mathrm{m} / \mathrm{s} \pm \mathrm{SD})$

Mean pressure gradient $(\mathrm{mmHg} \pm \mathrm{SD})$

Ejection fraction $(\mathrm{EF} \pm \mathrm{SD})$

$\log$ EuroScore

Comorbidities

Arterial hypertension $n(\%) \quad 42(100)$

CAD $n(\%)$

Diabetes mellitus $n(\%)$

Atrial fibrillation $n(\%)$

Renal insufficiency $n(\%)$

Peripheral vascular disease $n(\%)$

Previous myocardial infarction $n(\%)$

Pulmonary hypertension $n(\%)$

Previous stroke $n(\%)$

$A V A$ aortic valve area, $C A D$ coronary artery disease 